\section{ATAC-Seq Data for Genome-Wide Profiling of Transcription Factor Binding Sites in the Rice False Smut Fungus Ustilaginoidea virens}

\author{
Xiaoyang Chen, ${ }^{1}$ Hao Liu, ${ }^{1}$ Xiaolin Chen, ${ }^{1}$ Junbin Huang, ${ }^{1}$ Tom Hsiang, ${ }^{2}$ and Lu Zheng ${ }^{1, \dagger}$ \\ ${ }^{1}$ Hubei Key Laboratory of Plant Pathology, Huazhong Agricultural University, Wuhan 430070, \\ China \\ ${ }^{2}$ School of Environmental Sciences, University of Guelph, Guelph N1G 2W1, Canada
}

\begin{abstract}
Identification of transcription factor binding sites is one of the most important steps in understanding the function of transcription factors and regulatory networks in organisms. The assay for transposase accessible chromatin sequencing (ATAC-seq) is a simple protocol for detection of open chromatin that could be a powerful tool to advance studies of protein-DNA interactions. Although ATAC-seq has been used in systematic identification of cis-regulatory regions in animal and plant genomes, this method has been rarely applied in fungi. Here, we describe a valuable ATAC-seq resource in the genome of an economically important phytopathogen, the rice false smut fungus Ustilaginoidea virens. The ATAC-seq data of $U$. virens mycelia collected from potato sucrose broth (PSB) and PSB supplied with rice spikelet extract were both generated. This is the first genomewide profiling of open chromatin and transcription factor binding sites in $U$. virens.
\end{abstract}

Transcriptional regulation by a diverse array of transcription factors underpins the control of cellular development and virulence in phytopathogens. Identification of the transcription factors and their cognate transcription factor binding sites is one of the most important steps in understanding the function of transcription factors and regulatory networks. Rice false smut, caused by the pathogenic ascomycete Ustilaginoidea virens (teleomorph: Villosiclava virens), is one of the most devastating grain diseases in the majority of rice-growing areas of the world (Sun et al. 2020). Rice false smut not only causes yield loss but also threatens human or animal health by producing cyclopeptide mycotoxins in false smut balls (Koiso et al. 1994; Nakamura et al. 1994; Zhou et al. 2012). Genomic analyses of $U$. virens indicated that this fungus might contain over 300 transcription factors (Zhang et al. 2014). However, limited transcription factors have been experimentally verified, including UvHox2, UvCom1, and UVPRO1, which play important roles in development and virulence of this phytopathogen (Chen et al. 2020; Lv et al. 2016; Yu et al. 2019). As such, transcription factor binding sites in $U$. virens in genome-wide scale are not well understood.

${ }^{\dagger}$ Corresponding author: L. Zheng; luzheng@mail.hzau.edu.cn

Current address for L. Zheng: Hubei Key Laboratory of Plant Pathology, Huazhong Agricultural University, Wuhan 430070, China.

*The $e$-Xtra logo stands for "electronic extra" and indicates there is supplementary material published online.

The author(s) declare no conflict of interest.

Accepted for publication 5 February 2021.

\section{$e-X \operatorname{tra}^{*}$}

\section{Funding}

This work was supported by the National Natural Science Foundation of China (32072371), the National Key Research and Development Program (2017YFD0301400, 2016YFD0300700) and the

Fundamental Research Funds for the Central Universities of China (2662018JC051).

\section{Keywords}

ATAC-seq, binding site, transcription factor, Ustilaginoidea virens 


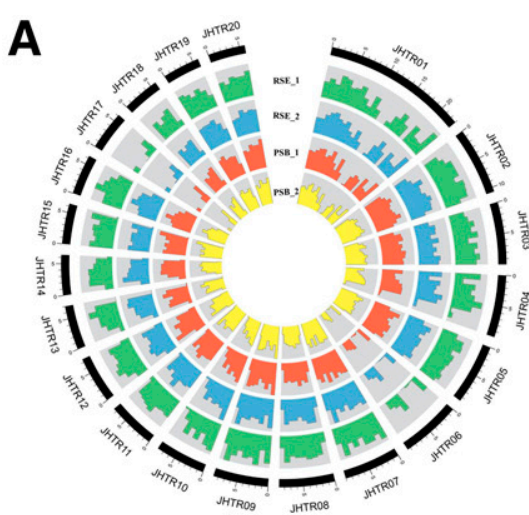

D

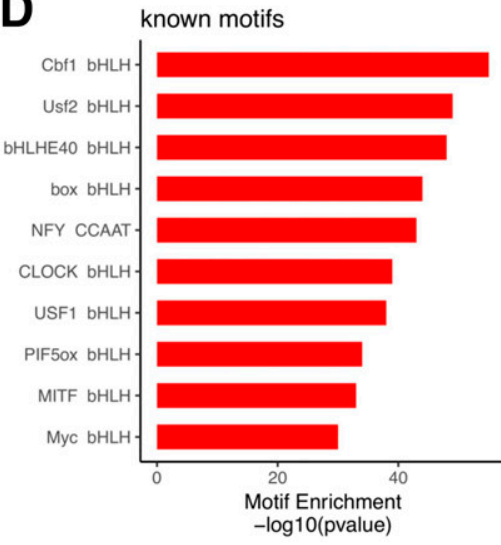

B
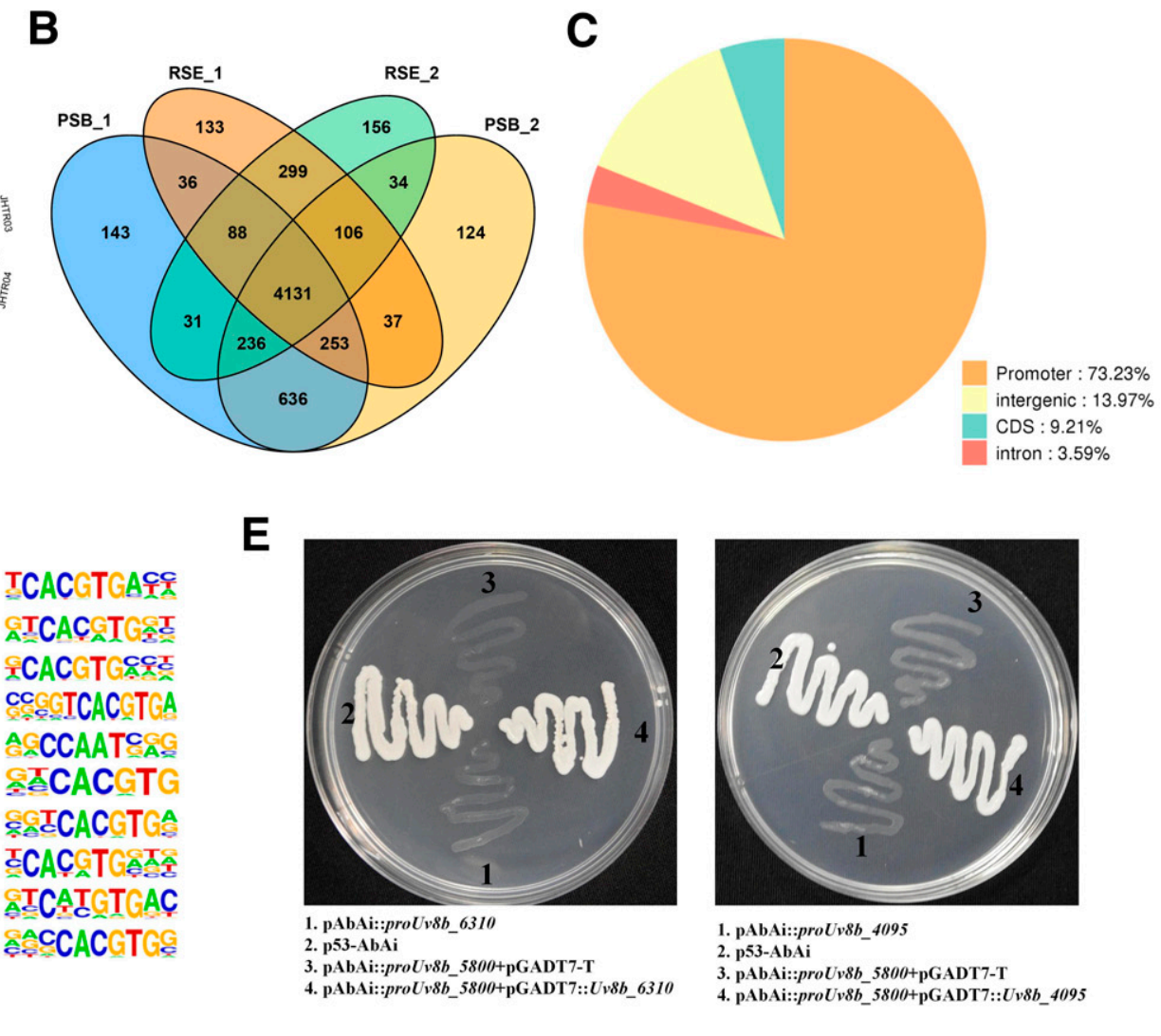

E

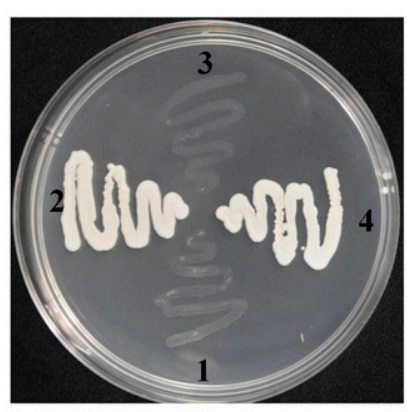

1. pAbAi::proUv8b_6310

2. p53-AbAi

3. pAbAi::proUv8b_5800+pGADT7-T

4. pAbAi::proUv8b_5800+pGADT7::Uv8b_6310

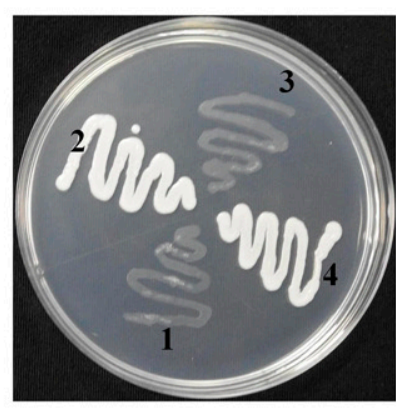

1. pAbAi::proUv8b_4095

2. p53-AbAi

3. pAbAi::proUv8b_5800+pGADT7-T

4. pAbAi::proUv8b_5800+pGADT7::Uv8b_4095

Fig. 1. Genome-wide profiling of transcription factor binding sites in Ustilaginoidea virens by ATAC-seq. A, Distribution of ATAC-seq peaks on the genome of $U$. virens. B, Venn diagram showing the overlap of TF-target genes defined by ATAC-seq peaks between the potato sucrose broth (PSB) and rice spikelet extract (RSE) treatments. C, Distribution of all ATAC-seq peaks in different regions of the U. virens genome. D, Logos of position weight coverage matrices from de novo-identified motifs occurring in nucleosome-free ATAC-seq peaks. E, Yeast one-hybrid assays showing that transcription factor Uv8b_5800 can bind to promoters of candidate target genes.

Experimental identification methods including DNase footprinting and electrophoretic mobility shift assays have, unfortunately, fallen far behind the rapid accumulation of genome sequences. Moreover, general high-throughput protocols such as ChIP-seq are costly and time-consuming. Recently, ATAC-seq (assay for transposase accessible chromatin sequencing) has been applied to systematically identify cis-regulatory regions and DNA footprints in animal and plant genomes (Lu et al. 2017; Wu et al. 2016). Chromatin structure plays a pivotal role in facilitating proper control of gene expression (Boyle et al. 2008; Felsenfeld 1992). Transcription factor binding of cis elements is often associated with accessible chromatin regions (Lu et al. 2017). Therefore, the ability to identify these accessible regions throughout a genome will advance the understanding of the relationships among transcription factor binding, chromatin status, and regulation of gene expression. In ATAC-seq, a bacterial transposase, Tn5, that inserts a short fragment of DNA (a transposon) into another molecule of DNA is used (Buenrostro et al. 2013). Since Tn5 is unable to access DNA that is either bound by nucleosomes or has strongly bound transcription factors, it integrates transposons preferentially into accessible or open chromatin (Li et al. 2019). Thus, ATAC-seq can uncover open chromatin regions in which transcription factors might bind. The technology of ATAC-seq has been rarely applied in fungi, such as yeast and mushroom Sparassis latifolia (Hendrickson et al. 2018; Yang et al. 2020). However, this technology has not been used in plant-pathogenic fungi.

In this study, we used ATAC-seq to reveal active regulatory regions in $U$. virens. The strain $U$. virens HWD-2, which was isolated from infected spikelets in Wuhan, China (Jia et al. 2015), was used in ATAC-seq experiments. ATAC-seq was performed according to the previously described protocols (Wu et al. 2016). Approximately $0.5 \mathrm{~g}$ of mycelia grown in potato sucrose broth (PSB) or mycelia cultured in PSB supplied with $2 \%$ (wt/vol) rice spikelet extract (RSE) were collected and were immediately ground in $2 \mathrm{ml}$ of prechilled lysis buffer (15 mM 
Table 1. Summary statistics of transcription factor interaction pairs probed by ATAC-seq in Ustilaginoidea virens

\begin{tabular}{|c|c|c|}
\hline \multirow[b]{2}{*}{ Type of transcription factor ${ }^{a}$} & \multicolumn{2}{|c|}{ Number of } \\
\hline & Genes $^{b}$ & Interaction pairs $^{c}$ \\
\hline $\mathrm{Zn}_{2} \mathrm{Cys}_{6}$ & 73 & 3,430 \\
\hline Winged helix repressor DNA-binding & 39 & 6,617 \\
\hline Nucleic acid-binding, OB-fold & 35 & 4,945 \\
\hline $\mathrm{C}_{2} \mathrm{H}_{2}$ zinc finger & 32 & 2,385 \\
\hline Homeodomain-like & 20 & 2,585 \\
\hline HMG & 10 & 1,406 \\
\hline Zinc finger, DHHC-type & 10 & 990 \\
\hline Zinc finger, CCHC-type & 8 & 3,980 \\
\hline bZIP & 8 & 1,047 \\
\hline GATA type zinc finger & 6 & 503 \\
\hline Heteromeric CCAAT factors & 6 & 1,253 \\
\hline Transcription factor jumonji & 4 & 286 \\
\hline bHLH & 4 & 512 \\
\hline Transcription factor TFIIS & 4 & 381 \\
\hline Zinc finger, MIZ-type & 3 & 554 \\
\hline APSES & 3 & 102 \\
\hline MADS-box & 2 & 42 \\
\hline Homeobox & 2 & 126 \\
\hline p53-like transcription factor & 2 & 293 \\
\hline Zinc finger, LSD1-type & 2 & 64 \\
\hline Zinc finger, Rad18-type putative & 2 & 199 \\
\hline AT-rich interaction region & 1 & 195 \\
\hline Lambda repressor-like, DNA-binding & 1 & 69 \\
\hline CCR4-Not complex component, Not1 & 1 & 208 \\
\hline RFX DNA-binding domain & 1 & 91 \\
\hline Zinc finger, GRF-type & 1 & 42 \\
\hline Zinc finger, NF-X1-type & 1 & 18 \\
\hline DDT & 1 & 82 \\
\hline YL1 nuclear protein & 1 & 268 \\
\hline Bromodomain transcription factor & 1 & 219 \\
\hline Single-stranded DNA-binding transcriptional regulator & 1 & 160 \\
\hline
\end{tabular}

a Transcription factor type was classified based on the method of Zhang et al. 2014.

b Number of transcription factors per family in U. virens.

c Number of transcription factor interaction pairs in $U$. virens.

Tris- $\mathrm{HCl}, \mathrm{pH} 7.5,20 \mathrm{mM} \mathrm{NaCl}, 80 \mathrm{mM} \mathrm{KCl}, 0.5 \mathrm{mM}$ permine, $5 \mathrm{mM} \beta$-mercaptoethanol [2-ME], $0.2 \%$ TritonX-100). The total mixture was filtered twice with Miracloth and were then loaded onto the surface of $2 \mathrm{ml}$ of dense sucrose buffer $(20 \mathrm{mM}$ Tris- $\mathrm{HCl}, \mathrm{pH} 8.0,2 \mathrm{mM}$ $\mathrm{MgCl}_{2}$, $2 \mathrm{mM}$ EDTA, $15 \mathrm{mM}$ 2-ME, $1.7 \mathrm{M}$ sucrose, 0.2\% TritonX-100) in a 10-ml Falcon tube. The mixture containing nuclei was centrifuged at 2,200 $\times g$ at $4^{\circ} \mathrm{C}$ for $15 \mathrm{~min}$ and the pellet was resuspended in $500 \mu \mathrm{l}$ of prechilled lysis buffer. The nuclei were pelleted by centrifugation at $1,000 \times g$ at $4^{\circ} \mathrm{C}$ for $10 \mathrm{~min}$. The quality of nuclei was checked under microscope by 4',6-diamidino-2-phenylindole staining. Crude nuclei were resuspended in the transposition reaction mix (Mix $25 \mu \mathrm{l}$ of reaction buffer, $2.5 \mu \mathrm{l}$ of Nextera Tn5 transposase, and 22.5 $\mu \mathrm{l}$ of nuclease free $\mathrm{H}_{2} \mathrm{O}$ ) and were incubated at $37^{\circ} \mathrm{C}$ for $30 \mathrm{~min}$. Qiagen MinElute PCR purification kit was used to purify the DNA after transposition. The DNA was amplified using NEBNext High-Fidelity 2xPCR master mix for 10 to 15 cycles (Lu et al. 2017). Amplified libraries were purified by the Qiagen MinElute PCR purification kit. The library was eluted in $20 \mu \mathrm{l}$ of elution buffer (10 mM Tris buffer, $\mathrm{pH}$ 8.0). The quality of each purified library was assessed using Bioanalyzer and Q-bit. The libraries were multiplexed and were then sequenced using an Illumina HiSeq X Ten platform with 150-bp paired ends by Igenebook Biotechnology Co., Ltd. (Wuhan, China). This experiment was conducted twice.

The adapter and low-quality reads of raw data were filtered out using Trimmomatic (version 0.38) (Bolger et al. 2014), resulting in approximately 28.6 to 49.5 million reads for each treatment. The filtered read data were mapped using Hisat2 software (version 2.0.1) (Kim et al. 2015) to the reference U. virens genome (National Center for Biotechnology Information [NCBI] database) (Zhang et al. 2014) with mitochondrial and chloroplast sequences removed. The peak position information on the genome and peak area sequence information 
were scanned by MACS (version 2.1.0) (Zhang et al. 2008). A total of 7,089 and 7,081 peaks were identified in the two PSB replicate treatments, while 7,372 and 7,303 peaks were detected in the two RSE replicate treatments (Fig. 1A). The open chromatin ATAC-seq peaks totally targeted 6,443 genes on the $U$. virens genome, and 4,131 genes were common to four independent experiments (Fig. 1B). A total of 299 target genes were found only in the RSE treatments but not in the PSB treatments (Fig. 1B). Of all putative peak regions identified using ATAC-seq, $73.23 \%$ of the peaks were located in promoter regions, $13.97 \%$ were located in intergenic regions, $9.21 \%$ were in coding sequence regions, and $3.59 \%$ were in intronic regions (Fig. 1C). We further applied motif discovery to search for candidate transcription factors that could bind these regions. DNA motifs were discovered using multiple expectation maximizations for motif elicitation (MEME, version 5.1.1) (Bailey et al. 2006) of the identified peaks (Fig. 1D). Based on motif sequences, we identified 553,907 transcription factor binding sites, 33,052 interaction pairs, and 285 transcription factors associated with regulation of $U$. virens (Table 1; Supplementary Table S1). We then randomly selected two interaction pairs (Uv8b_5800 and Uv8b_6310; Uv8b_5800 and Uv8b_4095) for yeast onehybrid verification. The results showed that Uv8b_5800 could interact with Uv8b_6310 or Uv8b_4095 (Fig. 1E), confirming the reliability of the data for predicted interaction pairs. Based on transcription factor binding motifs in the JASPAR database (Sandelin et al. 2004), 23 transcription factors, including six bZIP and 17 homeodomain transcription factors were selected for target-binding site (TBS) prediction in the $U$. virens genome. The TBS prediction analysis revealed that total 7,934 interaction pairs were detected for six bZIP transcription factors, while 4,135 interaction pairs were predicted for 17 homeodomain transcription factors. Compared with ATAC-seq data, TBS prediction showed more interaction pairs and $92.8 \%$ of the interaction pairs in TBS prediction analysis were the same as those in ATACseq, suggesting that ATAC-seq might provide more precise TBS identification. Hence, the use of ATAC-seq based open chromatin profiling combined with motif discovery provided a valuable resource to identify functional genomic regulatory regions, master regulators, and gene regulatory networks controlling complex in vivo processes in $U$. virens.

All raw and processed data files described in this work were deposited in the $\mathrm{NCBI}$ Sequence Read Archive (SRA) under accession number PRJNA643907. The $U$. virens strain HWD-2 was preserved in the China Center for Type Culture Collection (Wuhan, China) under accession number CCTCC M 2011023.

\section{Author-Recommended Internet Resources}

JASPAR database: http://jaspar.genereg.net

NCBI database: https://www.ncbi.nlm.nih.gov/nuccore/JHTR00000000

\section{Literature Cited}

Bailey, T. L., Williams, N., Misleh, C., and Li, W. W. 2006. MEME: Discovering and analyzing DNA and protein sequence motifs. Nucleic Acids Res. 34:W369-W373.

Bolger, A. M., Lohse, M., and Usadel, B. 2014. Trimmomatic: A flexible trimmer for Illumina sequence data. Bioinformatics 30:2114-2120.

Boyle, A. P., Davis, S., Shulha, H. P., Meltzer, P., Margulies, E. H., Weng, Z., Furey, T. S., and Crawford, G. E. 2008. High-resolution mapping and characterization of open chromatin across the genome. Cell 132:311-322.

Buenrostro, J. D., Giresi, P. G., Zaba, L. C., Chang, H. Y., and Greenleaf, W. J. 2013. Transposition of native chromatin for fast and sensitive epigenomic profiling of open chromatin, DNA-binding proteins and nucleosome position. Nat. Methods 10:1213-1218.

Chen, X., Hai, D., Tang, J., Liu, H., Huang, J., Luo, C., Hsiang, T., and Zheng, L. 2020. UvCom1 is an important regulator required for development and infection in the rice false smut fungus Ustilaginoidea virens. Phytopathology 110:483-493.

Felsenfeld, G. 1992. Chromatin as an essential part of the transcriptional mechanism. Nature 355:219-224.

Hendrickson, D. G., Soifer, I., Wranik, B. J., Kim, G., Robles, M., Gibney, P. A., and Mclsaac, R. S. 2018. A new experimental platform facilitates assessment of the transcriptional and chromatin landscapes of aging yeast. eLife 7:e39911.
Jia, Q., Lv, B., Guo, M. Y., Luo, C. X., Zheng, L., Hsiang, T., and Huang, J. B. 2015. Effect of rice growth stage, temperature, relative humidity and wetness duration on infection of rice panicles by Villosiclava virens. Eur. J. Plant Pathol. 141:15-25.

Kim, D., Langmead, B., and Salzberg, S. L. 2015. HISAT: A fast spliced aligner with low memory requirements. Nat. Methods 12:357-360.

Koiso, Y., Li, Y., Iwasaki, S., Hanaoka, K., Kobayashi, T., Sonoda, R., Fujita, Y., Yaegashi, H., and Sato, Z. 1994. Ustiloxins, antimitotic cyclic peptides from false smut balls on rice panicles caused by Ustilaginoidea virens. J. Antibiot. (Tokyo) 47:765-773.

Li, Z., Schulz, M. H., Look, T., Begemann, M., Zenke, M., and Costa, I. G. 2019. Identification of transcription factor binding sites using ATAC-seq. Genome Biol. 20:45.

Lu, Z., Hofmeister, B. T., Vollmers, C., DuBois, R. M., and Schmitz, R. J. 2017. Combining ATAC-seq with nuclei sorting for discovery of cis-regulatory regions in plant genomes. Nucleic Acids Res. 45:e41.

Lv, B., Zheng, L., Liu, H., Tang, J., Hsiang, T., and Huang, J. 2016. Use of random TDNA mutagenesis in identification of gene UvPRO1, a regulator of conidiation, stress response, and virulence in Ustilaginoidea virens. Front. Microbiol. 7:2086.

Nakamura, K., Izumiyama, N., Ohtsubo, K., Koiso, Y., Iwasaki, S., Sonoda, R., Fujita, Y., Yaegashi, H., and Sato, Z. 1994. "Lupinosis"-like lesions in mice caused by ustiloxin, produced by Ustilaginoieda virens: A morphological study. Nat. Toxins 2:22-28. 
Sandelin, A., Alkema, W., Engström, P., Wasserman, W. W., and Lenhard, B. 2004. JASPAR: An open-access database for eukaryotic transcription factor binding profiles. Nucleic Acids Res. 32:D91-D94.

Sun, W. X., Fan, J., Fang, A. F., Li, Y. J., Tariqjaveed, M., Li, D. Y., Hu, D. W., and Wang, W. M. 2020. Ustilaginoidea virens: Insights into an emerging rice pathogen. Annu. Rev. Phytopathol. 58:3.1-3.23.

Wu, J., Huang, B., Chen, H., Yin, Q., Liu, Y., Xiang, Y., Zhang, B., Liu, B., Wang, Q., Xia, W., Li, W., Li, Y., Ma, J., Peng, X., Zheng, H., Ming, J., Zhang, W., Zhang, J., Tian, G., Xu, F., Chang, Z., Na, J., Yang, X., and Xie, W. 2016. The landscape of accessible chromatin in mammalian preimplantation embryos. Nature 534:652-657.

Yang, C., Ma, L., Xiao, D., Ying, Z., Jiang, X., and Lin, Y. 2020. Integration of ATAC-Seq and RNA-seq identifies key genes in light-induced primordia formation of Sparassis latifolia. Int. J. Mol. Sci. 21:185.

Yu, J., Yu, M., Song, T., Cao, H., Pan, X., Yong, M., Qi, Z., Du, Y., Zhang, R., Yin, X., and Liu, Y. 2019. A homeobox transcription factor UvHOX2 regulates chlamydospore formation, conidiogenesis, and pathogenicity in Ustilaginoidea virens. Front. Microbiol. 10:1071.

Zhang, Y., Liu, T., Meyer, C. A., Eeckhoute, J., Johnson, D. S., Bernstein, B. E., Nusbaum, C., Myers, R. M., Brown, M., Li, W., and Liu, X. S. 2008. Modelbased analysis of ChIP-Seq (MACS). Genome Biol. 9:R137.

Zhang, Y., Zhang, K., Fang, A., Han, Y., Yang, J., Xue, M., Bao, J., Hu, D., Zhou, B., Sun, X., Li, S., Wen, M., Yao, N., Ma, L. J., Liu, Y., Zhang, M., Huang, F., Luo, C., Zhou, L., Li, J., Chen, Z., Miao, J., Wang, S., Lai, J., Xu, J. R., Hsiang, T., Peng, Y. L., and Sun, W. 2014. Specific adaptation of Ustilaginoidea virens in occupying host florets revealed by comparative and functional genomics. Nat. Commun. 5:3849.

Zhou, L., Lu, S., Shan, T., Wang, P., Sun, W., Chen, Z., and Wang, S. 2012. Chemistry and biology of mycotoxins from rice false smut pathogen. Pages 109-130 in: Mycotoxins: Properties, Applications and Hazards. B. J. Melborn, and J. C. Greene, eds. Nova Science Publishers, New York. 